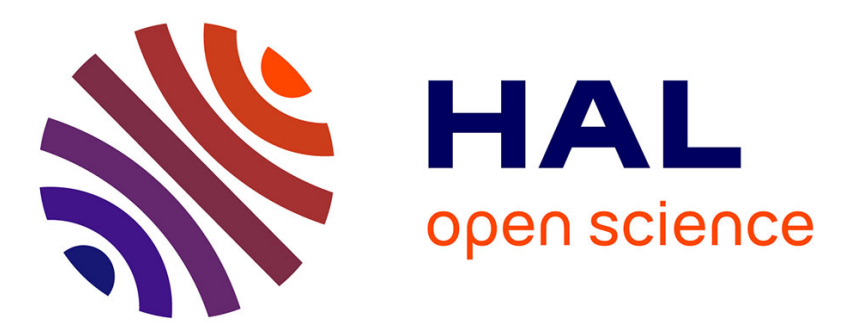

\title{
A Hierarchical System for Effective Coordination of Available-To-Promise Logic Mechanisms
}

\author{
Ioannis T. Christou, Stavros Ponis
}

\section{To cite this version:}

Ioannis T. Christou, Stavros Ponis. A Hierarchical System for Effective Coordination of AvailableTo-Promise Logic Mechanisms. International Journal of Production Research, 2009, 47 (11), pp.30633078. 10.1080/00207540701810786 . hal-00513018

\section{HAL Id: hal-00513018 \\ https://hal.science/hal-00513018}

Submitted on 1 Sep 2010

HAL is a multi-disciplinary open access archive for the deposit and dissemination of scientific research documents, whether they are published or not. The documents may come from teaching and research institutions in France or abroad, or from public or private research centers.
L'archive ouverte pluridisciplinaire HAL, est destinée au dépôt et à la diffusion de documents scientifiques de niveau recherche, publiés ou non, émanant des établissements d'enseignement et de recherche français ou étrangers, des laboratoires publics ou privés. 


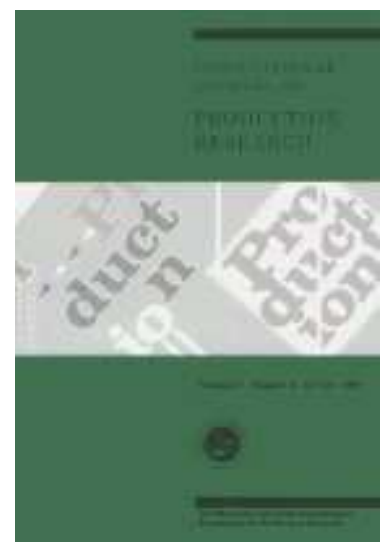

\section{A Hierarchical System for Effective Coordination of Available-To-Promise Logic Mechanisms}

\begin{tabular}{|r|l|}
\hline Journal: & International Journal of Production Research \\
\hline Manuscript ID: & TPRS-2007-IJPR-0753.R1 \\
\hline Manuscript Type: & Original Manuscript \\
\hline $\begin{array}{r}\text { Date Submitted by the } \\
\text { Author: }\end{array}$ & 24-Oct-2007 \\
\hline Keywords: List of Authors: & $\begin{array}{l}\text { Christou, Ioannis; Athens Information Technology } \\
\text { Ponis, Stavros; National Technical University of Athens, Mechanical } \\
\text { Engineering }\end{array}$ \\
\hline ERODUCTION PLANNING, AGGREGATE PLANNING, OPTIMIZATION, \\
\hline Keywords (user): & Available-To-Promise, Customer Order Decoupling Point \\
\hline
\end{tabular}

\section{ScholarONE \\ Manuscript Central}




\section{A Hierarchical System for Effective Coordination of}

\section{Available-to-Promise Logic Mechanisms}

Ioannis T. Christou

Athens Information Technology

19Km Markopoulou Ave. Paiania 19002 GREECE

ichr@ait.edu.gr

phone: $+30-210-668-2725$

fax: $+30-210-668-2703$

Stavros Ponis

Faculty of Mechanical Engineering

National Technical University of Athens

Iroon Polytehniou 9, Athens 15773 GREECE

staponis@central.ntua.gr

phone: +30-210-772-2384

fax: $+30-210-772-3571$ 


\title{
A Hierarchical System for Effective Coordination of
}

\section{Available-to-Promise Logic Mechanisms}

\begin{abstract}
This paper aims to provide a combinatory approach towards addressing the advanced ATP problem, consisting of three deterministic optimization models that operate on both sides of the Customer Order Decoupling Point. The proposed approach is based on long term aggregate capacity reservation for periods when increased volatility is expected, while still obtaining production plans that meet the predefined and agreed customer service levels. The three optimization models together guide a system that helps manufacturers to optimally decide on ATP quantity and due date quoting on the basis of available manufacturing resources. To support this system, a prototype software module was designed and implemented in Java that loosely integrates with the popular Open Source ERP system Compiere2's databases and uses the Linear Programming solver QS-Opt to solve the models developed in this research. The system response times as evidenced in the experiments described in this paper are quite acceptable for real-world operations. The proposed solution of the ATP problem is of great value for all competitive and proactive organizations that need a practical tool to support, in the best possible way and in an almost real time fashion, their decision on whether to accept or decline an incoming customer order request. It is our belief that an integration of the proposed models into existing ERP systems will enhance their limited ATP functionality and provide management with a powerful decision support tool.
\end{abstract}

Keywords: Production Planning, Aggregate Planning, Optimization, ERP, AvailableTo-Promise, Customer Order Decoupling Point. 


\section{Introduction}

Rapid response to customer needs, high level of customer service and flexibility to handle uncertainties and fluctuations in both demand and supply are becoming strategic differentiators in the modern marketplace. Organizations that want to achieve these benchmarks require sophisticated approaches to conduct order promising and fulfillment, especially in today's high mix - low volume production environment (Zhao et al., 2005). Available-to-promise (ATP) is such an approach, based on customer prioritization and capacity management in order to align the right inventory with the right customer for the right promise date (Cecere et al., 2005).

The concepts of Available-To-Promise (ATP) originate from a set of business practices that were eventually captured in the Association of Operations Management dictionary (APICS, 1987) as the method whereby a firm examines its available finished goods inventory, in order to agree on the quantity and promise a due date against a customer order request. A more recent definition by Gartner (2004), states that ATP is the uncommitted portion of a company's inventory or planned production, a figure that is frequently calculated from the Master Production Schedule (MPS) and is maintained as a tool for order promising. Ball et al. (2004) observed that the purpose of ATP is to operate on the boundary of push and pull-based controls. According to their approach, ATP problems are classified as being either 'push' or 'pull' based. The notions of push and pull are to be understood at the strategic level (Hopp \& Spearman 2001). At this strategic level, Ball et. al. define push as the set of mechanisms for production planning and distribution based on forecasts, whereas pull is defined as those mechanisms that are executed in response to customer orders. This view is consistent with Hopp and Spearman's definition of strategic push/pull (Hopp \& Spearman 2004), and in that sense exactly we shall use these concepts.

In this paper, we argue that a competitive and proactive organization should make every possible effort to combine the advantages of both pull and push based controls into an optimal interplay on the verges of both sides of the Customer Order Decoupling Point (CODP). The 
CODP separates the part of the supply chain geared towards directly satisfying customer orders from the part of the supply chain based on planning (Mason-Jones et al., 2000; Ball et al. 2004). In other words, CODP occurs at the point in the logistics chain where real demand meets the plan. To support this argument, in this paper, we propose a combinatory approach towards addressing the advanced ATP problem. We introduce a three-stage system which coordinates long-term planning decisions -made necessarily using forecasts- with short-term or daily sales \& operations scheduling activities -which operate upon actual order requests. Each stage in our system is controlled by a mathematical programming problem, whose solution provides the decision variables needed at that stage. The set of optimal controls of the first stage is required input for both the second and third stages, whereas the optimal controls for the second stage are part of the input of the final third stage, as will be explained in detail in the subsequent sections. The mathematical models of each stage necessarily differ with respect to the time granularity upon which they operate as well as in their complexity, but together provide a very simple and efficient coordination mechanism for manufacturing operations.

The remainder of this paper is organised as follows: In the next section a brief review of existing ATP literature is presented followed by the description of our proposed system's inputs and parameters. Next, the three optimization models for each stage of the system are formulated and thoroughly described supported by the necessary system workflow in the form of an algorithmic procedure. Thereupon, the applicability of the approach and time performance of the third and final, real-time stage solution is tested and evaluated using real life data from two large Greek manufacturers in the Food and Beverage industry. Finally, in the last section, conclusions, open issues and further research directions of our study are briefly discussed.

\section{Literature Review}

The relevant ATP literature can be broadly subdivided into textbook and management oriented publications as well as research papers introducing ATP methods in regard to 
specific practical ATP applications (Pibernik, 2005). Nevertheless, publications that provide mathematical models for quantity and due date promising are scarce. One of the first research efforts of that kind is traced back in the end of the previous century, when Taylor et al. (1999) proposed a finite capacity promising methodology that supported a continuous monitoring of the production capacity in order to fulfill additional incoming customer orders.

Ervolina et al. (2001), studied deterministic push-based ATP scheduling models for CTO (Configure-To-Order) systems with multiple products, components and time periods. Their work is based on the resource allocation software engines developed at IBM Research, and provides an important part of the foundation for the research developed in this paper. They do not provide any computational results.

One year later, Chen et al. (2002) consider a rolling horizon ATP pull-based problem for a Configure-to-Order (CTO) product. In their model, orders are batched over some prespecified period of time, after which order commitment dates and the production schedule are obtained by solving a multi-period mixed-integer program. In an earlier work, the same authors (Chen et al., 2001) consider a similar problem where customer due dates are flexible and a penalty for allowing component inventory levels to drop below a pre-specified reserve level is charged at the end of each batching interval.

Pull-based ATP provides the advantage of being responsive to disparities between actual and forecasted demand. In doing so pull based ATP scheduling repeatedly allocates resources after demand is realized. The notions of allocated ATP are more explicitly detailed in Kilger et al. (2005), where they draw heavily from concepts used for yield management in the airline industry (Smith et. al., 1992). Kilger et al. (2005) describe ATP as working along three major dimensions: customer, product and time. They define hierarchies along each of these dimensions and use a simple search procedure to find available-to-promise quantities. No optimization model is employed in their work.

Finally, a detailed study of ATP using deterministic optimization models is presented in Zhao et al. (2005), where the authors present a MIP model tailored to the specific requirements of ATP for an electronic product at Toshiba. In their efforts they take into 
account due date violations rules, manufacturing orders, production capability and capacity. The results were very promising in that they were able to simultaneously optimize both due date violations and inventory holding costs.

\section{A Hierarchical 3-Stage System for ATP Logic Mechanisms Coordination}

From a business point of view, ATP should be the set of processes that allow the company to decide in the best possible way, whether to accept or decline a customer order request, and to (optionally) best negotiate the request fulfillment's due date. These processes should be fast enough so as to allow sales personnel to respond to such requests in timeframes that are deemed acceptable by the customer. Most importantly, the ATP function should be properly aligned with the company's business model, practices or other hard issues such as production capacity or product life-time constraints.

Typical inputs that a business takes into account when considering whether or not to accept a customer order are the, a) customer order request data, b) customer significance, which most of the times is based on an $\mathrm{ABC}$ analysis classification of their key characteristics such as profitability or sheer size of their account (Christopher, 2005), c) long-term aggregate planning horizon and the decomposition of each aggregate period into fine-grain periods, d) existing current product demand forecasts for the planning horizon, e) production and distribution schedules, f) inventory levels for each warehouse and depot of the company and the associated geographic considerations and rules, g) existing promised orders and order details, h) factory capacity \& personnel work-schedules, i) raw materials and semi-finished goods inventories, together with BOM (Bill-of-Material) information for each product, $\mathrm{j}$ ) procurement schedules (raw material availability plans), k) MPS data including scheduled down-times according to maintenance policy, 1) product profitability details and m) the company business rules relating to service levels for each product. A business rule could also indicate that when a request cannot be fulfilled by the due date requested, the system should respond with another proposed later date. 


\subsection{System Modelling}

We propose a 3-stage system for the efficient coordination of ATP logic mechanisms. Each stage comprises a problem to be solved, formulated as an optimization model. The solutions of these problems coordinate the push and pull control practices as described earlier, as well as long-term planning decisions with sales and operations scheduling processes. ATP is viewed as the problem of deciding whether to accept a customer order request given the available inventory and planned production plus the remaining production capacity and the business rules concerning covering demand from certain customer demand classes, for given products and for a given time window. Whenever there is sufficient inventory allocated to a given customer for a certain product for a given time-period, the ATP problem becomes a simple search problem in the company warehouses and depots for the appropriate amounts of product requested. However, when the allocated inventory is not sufficient to cover an order request, there is still the possibility of modifying the production schedule (by utilizing "reserved" capacity and resources) to cover the extra demand. The business notion of 
reserving extra capacity for periods of high demand is thoroughly described in Christou et al., (2007).

The models together with some straight-forward search algorithms decide whether to accept a customer order, and if so, how to select inventory and possibly modify the production schedule to satisfy all committed customer orders. In doing so, the original aggregate long-term and medium-term plans of the company, remain unchanged. The models we formulate operate on different time-granularities on inventory and production controls. More specifically, at the push-control level, the Multi-Commodity Aggregate Production Planning (MCAP) model provides production plans to meet aggregate product demand for each aggregate period. Then, the Demand $\underline{\text { Allocation }} \underline{\text { ATP }}$ (DAATP) model provides a way to ration the aggregate production of a period among fine-grain level periods and among customer demand classes in a way to maximize company profits. Finally, the MultiCommodity Fine-grain Production Planning (MCFP) problem together with traditional allocated ATP search procedures operates at the pull-control level of actual sales and MTO mechanisms.

The assumptions we introduce in our models, are based on two real-world case studies drawn from the food and beverage industry. Both companies adopt traditional Make-To-Stock processes for certain products together with Make-To-Order practices for other products as well as to better react to unpredictable volatility in customer demand. On top of that, these companies carry the extra burden of producing products that have short life-times and cannot be kept in high inventories, a policy that would create a substantial risk of products perishing while in stock. Finally, specific priorities in the long-term planning process were set forth by the manufacturers that present a high degree of referentiality and can accommodate business scenarios by other manufacturers in the same industry.

\subsection{Stage 1: Multi-Commodity Aggregate Production Planning (MCAP Problem)}

Regarding aggregate production planning, we assume a multi-factory setting, where each factory has multiple production lines. Each line $\ell$ can produce a set of products at a particular 
rate measured in product units per hour where a product unit could be a package of 6 , or 1 Kg. of finished material etc. Each product has a limited life-time that starts the moment it is produced during which it may reach the downstream customer. There is a finite aggregate forecasting horizon for each period of which there is a forecast of total demand for each product. As is the practice in many companies in the foods and beverages industries, budgetary and planning concerns dictate a desired number of shifts for each line during each period, but this is not a hard constraint and can be viewed as an attempt to "reserve" line capacity during or immediately before periods of high demand (holiday seasons, periods that will follow promotional activities such as advertising campaigns, penetration into new markets etc.). More information on the determination of manpower shift planning, reservation or allocation, can be found in Lagodimos and Leopoulos (2000), or Lagodimos and Paravantis (2006).

The inputs to the MCAP problem are as follows:

- $\quad M=\{1, \ldots \mathrm{M}\}-$ aggregate forecasting horizon (usually 12 month-period)

- $T(\pi)$ - total life-time in number of aggregate periods of product $\pi$

- $\Pi(\ell)-$ set of products line $\ell$ can produce

- $\quad r_{\ell}^{\pi}$ - rate of production of product $\pi \in \Pi(\ell)$ when produced in line $\ell$

- $\quad d_{i}^{\pi}$ - demand forecast for each product $\pi$ during each period $i \in M$

- $w_{i}^{\pi}$ - relative weight of product $\pi$ during each period $i \in M$ signifying the importance of no stock-outs for this product relative to the other products in the range of products the company manufactures in period $i$

- $\quad h_{i}^{\ell}$ - desired number of shifts for line $\ell$ during period $i$

- $\quad D_{i}^{\ell}$ - total (maximum) number of calendar days line $\ell$ can operate during period

- $\quad S$ - total number of shifts in a day (usually 3 )

- $t$ - number of hours in a shift (usually 8 ) 
The above numbers for shifts and calendar days do not include sub-periods during which a line is scheduled to be down for maintenance or any other reasons.

The decision variables for the MCAP problem are the following:

- $\quad x_{i, j}^{\pi, \ell}$ - quantities for production for line $\ell$, of product $\pi \in \Pi(\ell)$ in period $i$ to be sold in later period $j$

- $\quad a_{i}^{\ell}$ - number of total shifts to be used in period $i$ in line $\ell$

- $\quad y_{i}^{\ell}$ - number of extra shifts that will be required in period $i$ on line $\ell$

- $\quad o_{i}^{\ell, \pi}$ - number of hours each line will have to work every period to produce product $\pi \in \Pi(\ell)$

- $\quad s_{i}^{\pi}$ - excess demand for a product $\pi$ in a period $i$ that cannot be physically produced by the company

The Multi-Commodity Aggregate Production Planning problem (MCAP) determines the optimal quantities for production, along with the number of total shifts to be used in period $i$ in line $\ell$ with a mention of the extra shifts per period. It also determines the optimal number of hours each line will have to work every period to produce the various products. We formulate the MCAP problem as follows:

$$
\min _{x, y, a, o, s} \sum_{i \in M} \sum_{\ell \in L} \sum_{\pi \in \Pi(\ell)} \sum_{j=i}^{\min (M, i+T(\pi))} 2^{j-i} x_{i, j}^{\pi, \ell}+K^{*} \sum_{i \in M} \sum_{\ell \in L} y_{i}^{\ell}+J^{*} \sum_{i \in M} \sum_{\pi \in \Pi} w_{i}^{\pi} s_{i}^{\pi}
$$

subject to:

- Production-demand balance constraints given finite products' life-time:

$$
\sum_{\ell \in L(\pi)} \sum_{j=\max (1, i-T(\pi))}^{i} x_{j, i}^{\pi, \ell}+s_{i}^{\pi}=d_{i}^{\pi} \quad \forall i \in M, \pi \in \Pi
$$

- Line-rates and shift-induced capacity constraints: 
- Definition of extra shifts constraint:

$$
y_{i}^{\ell}=a_{i}^{\ell}-h_{i}^{\ell} \quad \forall \ell \in L, i \in M
$$

- Upper-bound of total shifts constraint and integrality of total shifts

$$
a_{i}^{\ell} \leq D_{i}^{\ell} S, a_{i}^{\ell} \text { Integer } \quad \forall i \in M, \ell \in L
$$

- Non-negativity constraints:

$$
\begin{array}{ll}
x_{i, j}^{\pi, \ell} \geq 0 & \forall \pi \in \Pi, \ell \in L(\pi), i \in M, j=i, \ldots, \min (i+T(\pi), M) \\
y_{i}^{\ell} \geq 0 & \forall i \in M, \ell \in L \\
s_{i}^{\pi} \geq 0 & \forall i \in M, \pi \in \Pi \\
o_{i}^{\ell, \pi} \geq 0 & \forall i \in M, \ell \in L, \pi \in \Pi
\end{array}
$$

Setting the quantities $K^{*}=2^{M} \sum_{\pi \in \Pi, i \in M} d_{i}^{\pi}, \quad J^{*}=K^{*}\left(\sum_{i \in M, \ell \in L}\left(D_{i}^{\ell} S-h_{i}^{\ell}\right)+1\right)$

guarantee that the MCAP problem has an optimal solution $\left(x^{*}, y^{*}, a^{*}, o^{*}, s^{*}\right)$ that among all points in the feasible set

1. minimize the quantity $\sum_{i \in M, \pi \in \Pi} w_{i}^{\pi} s_{i}^{\pi}$

2. minimize the number of shifts above the desired shifts needed to produce the quantities $d_{i}^{\pi}-\left(s^{*}\right)_{i}^{\pi}$

3. minimize inventory holding time and costs among all feasible points that minimize the last two terms of the objective function.

A proof of the above statement for a slightly simplified version of the MCAP problem appears in Christou et al., 2007. The arguments of the proof however remain unchanged for the above stated problem. The MCAP problem, as it is defined above, takes into account line production rates that vary depending on the product being produced and different number of 
calendar days in each period that each line can be operational. The stability and numerical robustness of the solution of the MCAP model have been experimentally proven in Christou et al. 2007, where it is clearly shown that the exponential coefficients of the first term -which guarantee the 3 properties of the solution of the MCAP problem mentioned above- of the objective function do not stress at all the capability of standard simplex algorithms to find the optimal solution in a few seconds for the size of the real-world problems of our case studies.

\subsection{Stage 2: Demand Allocation Available-To-Promise (DAATP Problem)}

The next model we propose in this approach is responsible for allocating products' inventory to demand (customer) classes, and it is inspired from the push-based ATP model presented in Ball et al. (2004). Furthermore, it takes into account product life-time constraints and the particularities of a non-homogeneous multi-line manufacturing setting. The model rations available raw materials and production capacity among a set of demand classes $K$ and operates over a finer-level time horizon than the aggregate time horizon of MCAP. These classes could be either flat or they could form a hierarchy. This finer-level horizon analyzes each aggregate period $i$ into sub-periods (usually with a grain of one week) $M^{\prime}(i)$ that for brevity, when not ambiguous, will be denoted simply by $M$ '. The process is illustrated in Figure 1.

\section{Take in Figure 1}

Figure 1: Production and Inventory Controls in Varying Time-Grain Windows

As in the MCAP model, the set of products is denoted by $\Pi$. There is a set of raw materials $R$.The results of the MCAP problem above provide the aggregate quantities of each product $\pi$ that should be produced in aggregate period $i$ to meet the demands of the planning horizon, obeying as best as possible the soft constraints on the number of shifts to be used in the production plan. These quantities are computed as 


$$
\hat{X}_{i}^{\pi}=\sum_{\ell \in L(\pi)} \sum_{j=i}^{\min (M, i+T(\pi))} x_{i, j}^{\pi, \ell}
$$

Now, from the BoM we have the quantity $b_{r, \pi}$ of raw material $r \in R$ that is required to build a unit of product $\pi$. And from Material Request Planning we have the amount $q_{r, i}$ of raw material $r$ that will become available in period $i$.

The input data of the Demand $\underline{\text { Allocation }} \underline{\text { ATP }}$ (DAATP) problem are the following:

- $\quad \bar{d}_{i}^{\pi, k}$ - forecasted upper bound of the demand for product $\pi$ for demand class $k$ in period $i$.

- $d_{i}^{\pi}$ - forecasted total demand for product $\pi$ in period $i$.

- $\quad v^{\pi, k}$ - per unit net revenue for demand for product $\pi$ from demand class $k$,

- $b_{r, \pi}$ - raw material $r \in R$ that is required to build a unit of product $\pi$.

- $q_{r, i}$ - amount of raw material $r$ that will become available in period $i$.

- $\quad c_{i}^{\pi} \quad-$ cost of producing a unit of product $\pi$ in period $i$.

- $h_{i}^{\pi}$ - cost of holding inventory of a unit of product $\pi$ in period $i$

- $\quad h_{i}^{\prime r} \quad$ - cost of holding inventory of a unit of raw material $r$ in period $i$.

- $\quad \hat{X}_{i}^{\pi}$ - quantity of product $\pi$ to be produced in aggregate period $i$.

- $\quad M^{\prime}$ - a fine-level planning horizon covering the aggregate period $i$.

The decision variables for the DAATP problem are the following:

- $\quad Y_{j, i}^{\pi, k}$ - quantity of product $\pi$ produced in period $j$ allocated to class $k$ in period $i \geq j$. 
- $Y_{i}^{\pi, k}-$ total quantity of product $\pi$ allocated to class $k$ in period $i$.

- $\quad I_{j, i}^{\pi}$ - inventory of product $\pi$ produced in period $j$ held in period $i \geq j$.

- $I_{i}^{\pi}$ - total inventory of product $\pi$ held in period $i$.

- $\quad J_{i}^{r}$ - inventory of raw material $r$ held in period $i$.

- $\quad X_{i}^{\pi}$ - quantity of product $\pi$ to be produced in fine-grain period $i$.

The push-based DAATP problem becomes the following Linear Program:

$$
\max _{Y, I, J, X} \sum_{i \in M^{\prime}} \sum_{k \in K} \sum_{\pi \in \Pi} v^{\pi, k} Y_{i}^{\pi, k}-\sum_{i \in M^{\prime}, \pi \in \Pi} h_{i}^{\pi} I_{i}^{\pi}-\sum_{i \in M^{\prime}, r \in R} h_{i}^{\prime r} J_{i}^{r}-\sum_{i \in M^{\prime}, \pi \in \Pi} c_{i}^{\pi} X_{i}^{\pi}
$$

subject to:

- Demand and availability limitations:

$\sum_{k \in K} Y_{i}^{\pi, k} \leq I_{i}^{\pi}+X_{i}^{\pi} \quad \forall i \in M^{\prime}, k \in K, \pi \in \Pi$

$\sum_{k \in K} Y_{i}^{\pi, k} \leq d_{i}^{\pi} \quad \forall i \in M^{\prime}, k \in K, \pi \in \Pi$

$Y_{i}^{\pi, k} \leq \bar{d}_{i}^{\pi, k} \quad \forall i \in M^{\prime}, k \in K, \pi \in \Pi$

- Product inventory balance subject to life-time constraints:

$$
\begin{aligned}
& I_{i}^{\pi}=\sum_{j=\max (1, i-T(\pi))}^{i-1} I_{j, i}^{\pi} \quad \forall i \in M^{\prime}, \pi \in \Pi \\
& Y_{i}^{\pi, k}=\sum_{j=\max (1, i-T(\pi))}^{i} Y_{j, i}^{\pi, k} \quad \forall i \in M^{\prime}, \pi \in \Pi, k \in K \\
& I_{j, i}^{\pi}=\left\{\begin{array}{cc}
X_{j}^{\pi}-\sum_{k \in K} \sum_{j^{\prime}=j}^{i-1} Y_{j, j^{\prime}}^{\pi, k} & j \leq i-1 \\
0 & \text { else }
\end{array}\right\} \forall i, j \in M^{\prime}, \pi \in \Pi
\end{aligned}
$$


- Material inventory balance:

$$
J_{i-1}^{r}+q_{r, i}=J_{i}^{r}+\sum_{\pi \in \Pi} b_{r, \pi} X_{i}^{\pi} \quad \forall i \in M^{\prime}, r \in R
$$

- Aggregate production requirements:

$$
\sum_{i^{\prime} \in M^{\prime}(i)} X_{i^{\prime}}^{\pi}=\hat{X}_{i}^{\pi} \quad \forall i \in M, \pi \in \Pi
$$

- Initialization and non-negativity

$$
I_{i, j}^{\pi} \geq 0, I_{j, 0}^{\pi}=p_{j}^{\pi}, J_{0}^{r}=q_{r, 0}, J_{i}^{r} \geq 0, X_{i}^{\pi} \geq 0, Y_{i, j}^{\pi, k} \geq 0
$$

The (sales) quantities $Y_{i}^{\pi, k}$ determined from the solution of the DAATP problem not only specify how to allocate -anticipated- demand during the current aggregate period, but also serve as ratios to allocate "reserved" lines capacities as we shall see in the formulation of the next model. We consider DAATP as a push control because as is the case with MCAP, it is also based on forecast demand as opposed to actual demand manifested by sales orders. Indeed, the model is dependent on the fundamental forecasted quantities $d_{i}^{\pi}, \bar{d}_{i}^{\pi, k}$ as well as the quantities $\hat{X}_{i}^{\pi}$ which are based on the aggregate demand forecasts.

DAATP is essentially the link between long-term planning and short-term sales and operations scheduling; MCAP provides long-term aggregate production levels, used by DAATP to compute the short-term allocated inventory quantities $Y_{i}^{\pi, k}$ so as to maximize profitability within the current aggregate period. These quantities, in conjunction with an appropriate rationing of the unused line capacities are passed as input to the final third stage's optimization problem, the Multi-Commodity Fine-grain Production Planning problem (MCFP) to determine whether to accept a new customer order request, or how to modify its requested due-date so as to be able to satisfy it. 


\subsection{Stage 3: Multi-Commodity Fine-grain Production Planning (MCFP Problem)}

MCFP operates at the pull-control level in the sense that it does not directly depend on demand forecasts, but executes upon actual order requests arrivals. In the case when customer classes do not form a tree hierarchy but instead form a flat partitioning of the total customer accounts, it accepts as input the allocated inventory $Y_{i}^{\pi, k}$ for each customer class per period per product, as computed by the solution of the DAATP model. As a second input the model accepts a set of pending customer orders; each customer order is a set of quadruples $\left(i, \pi, k, d_{i}^{\pi, k}\right)$ where $k$ is the customer class where the customer belongs. This set of open customer orders is denoted by CustOrder. The third input is the remaining unutilized possible number of shifts $u_{i}^{\ell}=D_{i}^{\ell} S-a_{i}^{\ell} \quad \forall i \in M, \ell \in L$ for the current aggregate period $i$, for each line, as determined by the MCAP problem solution. The fourth input is a decomposition $M^{\prime}(i)$ of the current aggregate period -i.e. the same time horizon used in the DAATP problem- and a rationing of the total number of currently unused hours $o_{i}^{\pi, k} \quad \forall \pi \in \Pi, i \in M^{\prime}, k \in K$ among customer demand classes $k \in K$ per product per period. The rationing of unused production hours per customer per product per period is executed in a way so that their percentage is the same as the allocated ATP determined by the solution of the DAATP problem.

To summarize, the MCFP problem inputs are then the following:

- $\quad Y_{i}^{\pi, k}$ - allocated inventory for each product per period per customer class

- CustOrder - set of pending customer orders expressed as tuples of the form $\left(i, \pi, k, d_{i}^{\pi, k}\right)$

- $\quad u_{i}^{\ell}$ - unutilized shifts during current aggregate period $i$ in line $\ell$

- $\quad M^{\prime}(i)$ - decomposition of current aggregate period $i$ into fine-grain periods

- $o_{i}^{\pi, k}$ - a rationing of the total number of currently unused hours among customer demand classes $k \in K$ per product per fine-grain period 
The MCFP problem determines whether a feasible schedule exists that will produce within the current aggregate period, all the product quantities identified in the customer order request within the fine-grain time period specified. The decision variables of the MCFP problem are the following:

- $\quad x_{i, j}^{\pi, \ell, k}$ - quantity of product $\pi$ to be produced in line $\ell \in L(\pi)$ during fine-grain period $i$ to be delivered in fine-grain period $j$ to customer class $k \in K$.

- $\quad e_{i}^{\ell, \pi, k}$ - the hours that line $\ell$ must operate in fine-grain period $i$, on product $\pi$ for customer $k \in K$.

The pull-based MCFP problem becomes the following Linear Program:

$$
\min _{x, e} \cdot \sum_{i \in M^{\prime}} \sum_{\ell \in L} \sum_{\pi \in \Pi(\ell)} \sum_{k \in K}\left(\sum_{j=i}^{\min \left(i+T(\pi), M^{\prime}\right)} 2^{j-i} x_{i, j}^{\pi, \ell, k}\right)
$$

subject to:

- Planned production and allocated inventory vs. demand constraints:

$$
Y_{i}^{\pi, k}+\sum_{\ell \in L(\pi)} \sum_{j=\max (1, i-T(\pi))}^{i} x_{j, i}^{\pi, \ell, k} \geq \sum_{(i, \pi, k) \in \text { Custorder }} d_{i}^{\pi, k} \quad \forall(i, \pi, k) \in \text { CustOrder }
$$

- Line-rate production balance constraints:

$$
\sum_{j=i}^{\min \left(i+T(\pi), M^{\prime}\right)} x_{i, j}^{\pi, \ell, k}=e_{i}^{\ell, \pi, k} r_{\ell}^{\pi} \quad \forall \ell \in L, i \in M^{\prime}, \pi \in \Pi(\ell), k \in K
$$

- Shift-induced upper bound on total hours each line may operate:

$$
\sum_{j \in M^{\prime}(i)} \sum_{\pi \in \Pi} \sum_{k \in K} e_{j}^{\ell, \pi, k} \leq u_{i}^{\ell} t \quad \forall i \in M, \ell \in L
$$

- Rationing of unused hours constraint:

$$
\sum_{\ell \in L(\pi)} e_{i}^{\ell, \pi, k} \leq o_{i}^{\pi, k} \quad \forall i \in M^{\prime},(\pi, k) \in \text { CustOrder }
$$

- Non-negativity constraints:

$$
x_{i, j}^{\pi, \ell, k} \geq 0 \quad e_{i}^{\ell, \pi, k} \geq 0
$$


Notice that the above problem -as opposed to the MCAP problem which is always feasible- may well be infeasible. This would be the case if there are not enough extra hours allocated for production of products from a customer in fine-grain periods $i \in M^{\prime}$. Should this case arise, the system workflow (discussed next) modifies the customer order request's due date to the earliest possible date within the current aggregate period that the order can be fulfilled, or eventually rejects the order if no such date can be found.

The combination of these three models as an integrated system can give optimal solutions to the ATP scheduling problem. The coordination of the three models is schematically shown in Figure 2.

Take in Figure 2

Figure 2: Coordination of MCAP/DAATP/MCFP Models

In the remainder of this section and to enhance the understanding of this paper, our approach is further explained in the form of a step-by-step application roadmap. The whole system workflow consists of the following discrete steps:

\section{System Workflow}

1. Solve the MCAP problem to determine next periods' aggregate production requirements based on the latest updates of market forecasts for the company's products.

2. Solve the DAATP problem to determine how to allocate current and planned product inventory among the current aggregate period's finer level time intervals and customer classes. 
3. Using the solution of the DAATP problem in Step 2, compute a customer/product/period cube that contains the allocated quantities of each product to each customer class in each fine-grain level period.

4. Using the same portion of the allocated products per customer class per period, allocate the total extra hours $o_{i}^{\pi}$ of unused capacity available during this aggregate period, to customer classes per period per product.

5. When a customer order request arrives, first check via simple search among the customer classes to which the customer belongs in a bottom-up fashion for available inventory in the periods up to the period requested. For any remaining product quantities that cannot be found in the inventory cube, proceed to Step 6.

6. Solve the MCFP problem to determine whether a production plan exists that can satisfy all current constraints and is able to provide the required remaining product quantities until the customer requested due-date. If such a plan exists, the order request is accepted and the appropriate book-keeping procedures are triggered to modify the production schedule to accommodate for the new order. If this is not the case then the order is modified by increasing its due-date by one fine-grain period and MCFP is run again, until either the problem becomes feasible or all fine-grain periods within the current coarsegrain period have been checked. In the first case, the new date is proposed as an alternative, while in the latter case, an alternative smaller order quantity is proposed based on the quantities computed in step 5.

From a business logic perspective, the above workflow reveals the fact that in order for our proposed approach to be feasible, the MCFP problem must be solved in almost real-time, thus providing sales force the opportunity to promptly respond to customer requests, in any occasion. To address this issue, real life data from the model's application in two Greek manufacturers operating in the food and beverage industry are presented. 
Food and beverages constitute an ideal case for this study because they are characterized by strong seasonality in demand and short product life-time constraints. The first case company is a beverage manufacturer (soft drinks, teas and juices) confronting a problem of 40 different final products, produced in 14 different lines over a long-term horizon of 12 months and short-term horizon of 4 weeks The products have life-times ranging from 2 weeks to 15 months. The major customer accounts (demand classes) are less than 50. The second company is a 'meat and sausages' manufacturer. In that case the problem that needs to be solved is more complex, involving 360 products, 17 production lines and 30 customer accounts. Each meat product has a nominal life-time less than two months from production date.

The solution of the MCAP and DAATP models is an off-line process that poses no serious constraints on the performance of the algorithms employed for their solution. Nevertheless, the MCAP problem, in both cases, is easily solved on the NEOS servers in a few seconds. Therefore, the challenge lies, as mentioned earlier, in solving the MCFP problem in an almost real-time manner.

At this point, it is of value to remind that, MCFP is a Linear Program with $\left(\frac{M^{\prime}\left(M^{\prime}+1\right)}{2}+M^{\prime}\right) \Pi L K_{\text {variables and }} O\left(M^{\prime}+1\right)+L M^{\prime} \Pi K_{\text {non-trivial constraints and }}$ $M^{\prime} \Pi L K\left(\frac{M^{\prime}+1}{2}+1\right)$ variable non-negativity constraints. The MCFP problem can be further accelerated if we take into account that all the orders entered in the system can be represented with at most $M^{\prime} \Pi K$ constraints. Indeed, every order to be considered is a set of quadruples of the $\left(i, \pi, k, d_{i}^{\pi, k}\right)$ form. Different orders for the same period, same product coming from the same customer class can be concatenated into one quadruple containing the sum of their individual demands.

In that setting and for the most complex case, the size of the problem can reach up to 300.000 variables and more than 100.000 constraints. Solving a problem of that magnitude on the NEOS servers using GAMS/BDMLP was not possible. However, solving the problem on 
a Pentium 4 (3.2 GHz) desktop, running Windows with 2GB of RAM using the QSOpt LP package takes less than 5 seconds, which allows a sales representative to respond in real-time to a customer placing an order request over the phone. Interestingly, the solution time using QSOpt's dual simplex method does not vary significantly under the five different scenarios CF60[a-e]. These scenarios are data sets representing identical problem size, but drastically different planning situations, ranging from almost unutilized lines for most periods to scenarios where most lines are busy most of the time, thereby having no free-time for new orders, thus leading to customer order request rejection. In detail, the results of a number of tests with some of the hardest data set configurations for the two manufacturers in our case study are summarized in the table I below.

Take in Table I.

Table I. Time Performance of the MCFP Problem Solution for Different Data Sets

\section{Conclusions and Further Directions}

In this paper, we presented a novel combinatory approach towards addressing the advanced ATP problem that operates on both sides of the CODP, thus transforming it from a barrier between push and pull based ATP approaches to a coordination mechanism between long-term decision making and customer order fulfillment. In doing so, three different optimization models have been elaborated that, tightly coupled together, guide a system which helps manufacturers in markets exhibiting significant variations in demand to decide, in the best possible way, on quantity and due date order promising.

The cornerstone of our approach is undoubtedly the idea of long-term aggregate capacity reservation for periods when increased volatility is expected, while still obtaining production plans that meet the customer service levels required by the company. This goal is achieved by an optimization problem (MCAP). Then, planned production and stocked inventories are rationed in an "Allocated ATP" style among customer classes by executing a linear problem 
(DAATP) which provides the solution on how to allocate the available ATP quantities. With that as an input, a separate straight-forward algorithm allocates reserved capacity among customer classes. Finally, order-driven activities are coordinated by the real-time solution of yet another linear program (MCFP), which decides on the ATP quantities and if needed, on what reserved production capacity to commit to a newly arrived customer order request.

In an attempt to field test our approach, we have ran our models with real-world data set sizes from two Greek manufacturing companies in the Food and Beverage Industry. We have found that solving the MCFP model with the dual simplex method as implemented in the freely available QSOpt package produces excellent results in terms of response time, allowing sales personnel to respond, in real-time, to a customer order request over the phone. The solutions obtained are optimal, with respect to the model solved, and they produce plans that minimize the time between the order due date and the production date, for the products included in the order and not already allocated or planned in inventory via the allocation mechanisms of DAATP.

Despite the attractiveness and practical value of our approach, a number of open issues still remain, such as the business workflow and practices that have to be adopted by a company in order to successfully support our approach and furthermore the level of human intervention on the system prompts and the final decision making, based on the previously decided workflow. Our experience from applying ATP scheduling processes in real life business applications has shown that often, system prompts are opposed to specific business practices or more flexible managerial decisions, a fact that most of the times leads to system depreciation. Studying these issues in more detail will enhance our understanding of the ATP problem and would likely yield much insight into the business models and practices that would make a company more capable of meeting its strategic goals such as profitability and high levels of customer service and satisfaction. 


\section{References}

APICS (1987), American Production and Inventory Control Society Dictionary, $6^{\text {th }}$ Ed., Falls Church, VA.

Applegate, D. Cook, W., Dash, S. and Mevenkamp, M. (2003), QSOpt Reference Manual, Dept. of Industrial Eng. Georgia Institute of Technology, Tech. Report.

Baker, K.R., Powell, S.G. and Pyke, D.F. (1990), “The performance of push and pull systems: a corrected analysis", International Journal of Production Research, Vol. 28 (9), pp.1731-6. Ball, M. O., Chen, C-Y. and Zhao, Z-Y. (2004), “Available To Promise”, in Simchi-Levi, D., Wu, S.D. and Shen, Z.M (eds): Handbook of Quantitative Supply Chain Analysis: Modeling in the E-Business Era, Springer's Intl. Series, NY. pp. 447-483.

Cecere L., Hofman D., Martin, R. and Preslan, L. (2005), The Handbook for Becoming Demand Driven, AMR Research Inc..

Chen, C-Y. Zhao, Z-Y. and Ball, M.O. (2001), “Quantity and Due-Date Quoting Available To Promise", Information Systems Frontiers, Vol. 3 No. 4, pp. 477-488

Chen, C-Y. Zhao, Z-Y. and Ball, M.O. (2002), "A Model for Batch Advanced Available To Promise", Production and Operations Management, Vol. 11 No. 4, pp. 424-440.

Chen-Ritzo, C.-H. (2006), Availability Management for Configure-To-Order Supply Chain Systems, PhD Thesis, College of Business Administration, Pennsylvania State University.

Christopher, M. (2005), Logistics and Supply Chain Management: Creating Value-Adding Networks, $3^{\text {rd }}$ Edition, Prentice-Hall, Harlow, UK.

Christou I. T., Lagodimos, A.G. and Lycopoulou, D. (2007), "Hierarchical Production Planning for Multi-Product Lines in the Beverage Industry", Journal of Production Planning \& Control, Vol. 18 No. 5, Jul. 2007

Ervolina T. and Dietrich, B. (2001), "Moving Toward Dynamic Available To Promise”, in Gass, P.I. and Jones, A.T. (eds), Supply Chain Management Practice \& Research: Status and 
Future Directions, R.H. School of Business, University of Maryland, Manufacturing Engineering Laboratory.

Hopp, W.J., and Spearman, M.L. (2001), “Factory Physics: Foundations of Manufacturing Management", McGraw-Hill, NY, NY.

Hopp W.J., and Spearman, M.L. (2004), “To Pull or Not to Pull: What is the Question?" Manufacturing \& Service Operations Management, Vol.6 (2), pp.133-148.

Kilger C. and Schneeweiss, L. (2005), "Demand Fulfillment and ATP”, in Stadtler H. and Kilger C. (eds.), Supply Chain Management and Advanced Planning: Concepts, Models, Software and Case Studies, Springer, Berlin, Germany.

Lagodimos A.G., and Leopoulos V. (2000), “Greedy Heuristic Algorithms for Manpower Shift Planning”, International Journal of Production Economics, Vol. 68, No 1, pp. 95-106. Lagodimos A.G., and Paravantis, J. (2006), "Improved Heuristic for Manpower Shift Planning with Modified Shift Priorities", Journal of Production Planning \& Control, Vol. 17 No. 3, pp. 301-310.

Mason-Jones R., Naylor B. and Towill, D.R. (2000), "Engineering the Leagile Supply Chain”, International Journal of Agile Management Systems, Vol. 2 No. 1, pp. 54-61.

Pibernik, R. (2005), “Advanced Available-to-promise: Classification, Selected Methods and Requirements for Operations and Inventory Management", Int. Journal of Production Economics, Vol. 93-94 No. 1, pp. 239-252.

Smith, B., Leimkuhler, J., Darrow, R. and Samuels, J. (1992), "Yield Management at American Air-Lines", Interfaces, Vol. 22 No. 1, pp 8-31.

Spearman, M. L., M. A. Zazanis (1992), "Push and pull production systems: Issues and comparisons", Operations Research, Vol.40, pp.521-532.

Taylor, S.G. and Plenert, G.J. (1999), "Finite Capacity Promising”, Production and Inventory Management Journal, Vo. 40 No. 3, pp. 50-56. 
Zhao, Z., Ball, M.O. and Kotake, M. (2005), “Optimization-based Available-To-Promise with

Multi-Stage Resource Availability", Annals of Operations Research, Vol. 135 No. 1, pp 6585. 


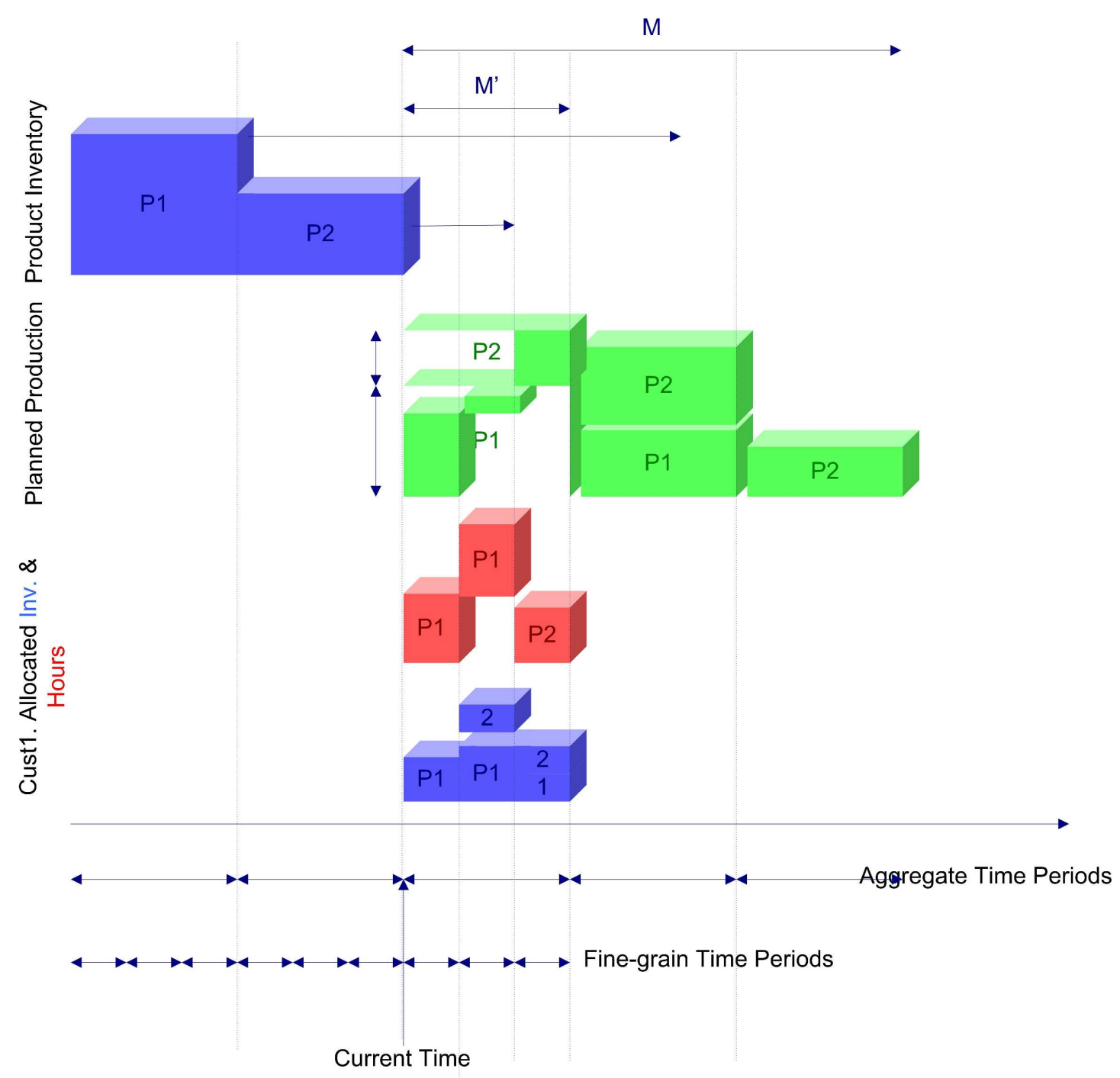

Figure 1: Production and Inventory Controls in Varying Time-Grain Windows 


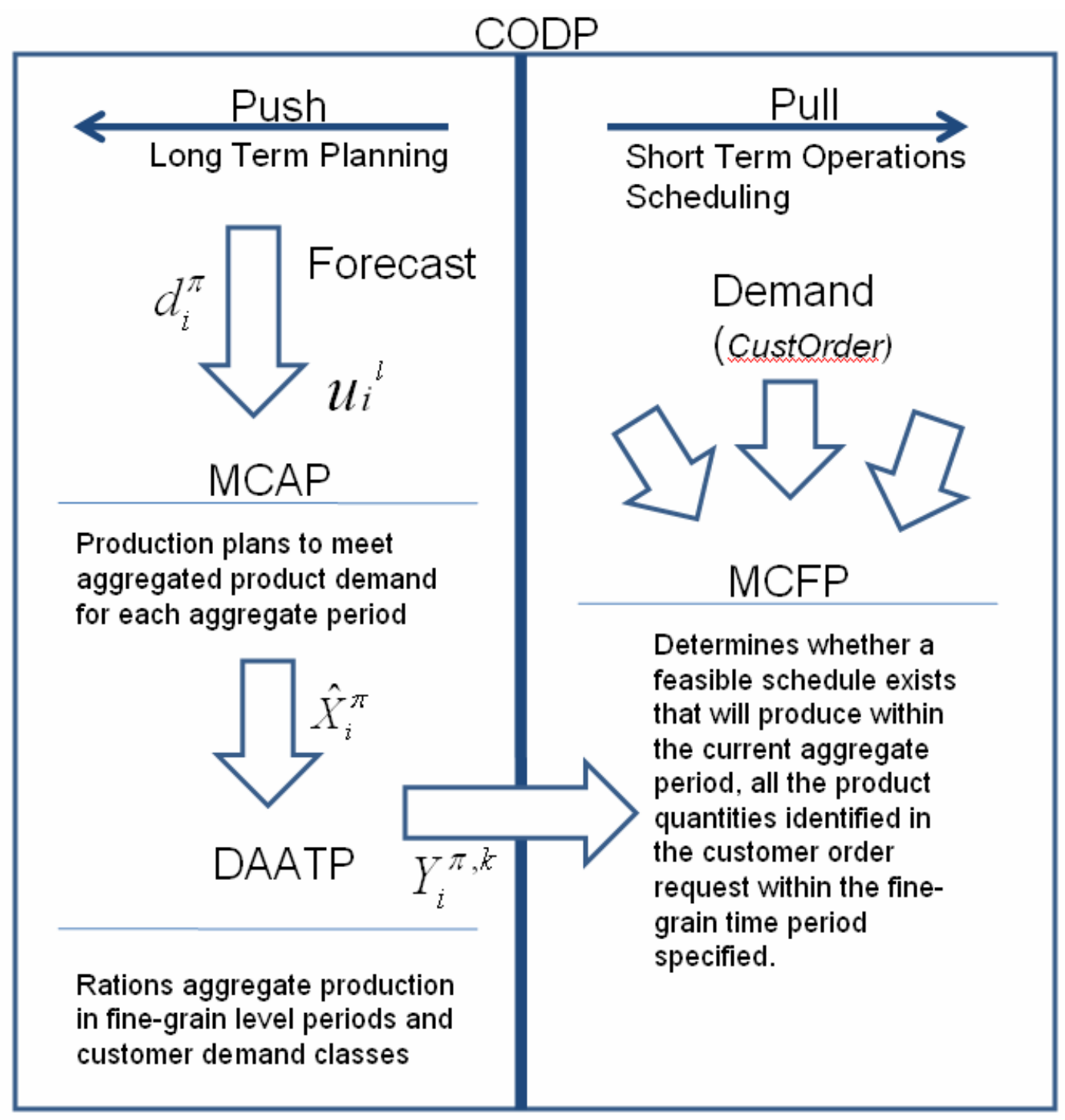

Figure 2: Coordination of MCAP/DAATP/MCFP Models 


\begin{tabular}{|ccccccc|}
$\begin{array}{c}\text { D Dataset } \\
\text { Name }\end{array}$ & $\begin{array}{c}\text { Number of } \\
\text { Lines }\end{array}$ & $\begin{array}{c}\text { Number of } \\
\text { Products }\end{array}$ & $\begin{array}{c}\text { Number of } \\
\text { Periods }\end{array}$ & $\begin{array}{c}\text { Number of } \\
\text { Customers }\end{array}$ & $\begin{array}{c}\text { MCFP } \\
\text { Runtime (in } \\
\text { seconds) }\end{array}$ \\
\cline { 7 - 7 } Ex7 & & 2 & & 4 & 2 & 0.8 \\
CF10 & 14 & 10 & 4 & 30 & 1.5 \\
CF60a & 14 & 60 & 4 & 30 & 5 \\
CF60b & 14 & 60 & 4 & 30 & 7 \\
CF60c & 14 & 60 & 4 & 30 & 5 \\
CF60d & 14 & 60 & 4 & 30 & 10 \\
CF60e & 14 & 60 & 4 & 30 & 15 \\
3E1 & 14 & 8 & 4 & 20 & 2 \\
\hline
\end{tabular}

